\title{
Manejo conservador de un traumatismo esplénico iatrogénico tras la inserción de un tubo de tórax
}

\author{
Conservative management in iatrogenic splenic trauma after chest tube insertion
} Natalia Gutiérrez-Corral' * , Isabel García-Bear', Carmen García-Gutiérrez² y Susana González-Sanmartino ${ }^{3}$
'Servicio de Cirugía General y Aparato Digestivo, Hospital Universitario San Agustín, Avilés; ${ }^{2} 2$ Servicio de Cirugía General y Aparato Digestivo,
Hospital Universitario Central de Asturias; 3Área de Radiodiagnóstico. Hospital Universitario Central de Asturias. Asturias, España

\section{Resumen}

Insertar un tubo torácico es una maniobra terapéutica de gran valor, pero no exenta de complicaciones. Nuestro objetivo es poner de manifiesto una nueva opción de tratamiento mediante técnicas radiológicas intervencionistas que eviten los riesgos de una cirugía en pacientes seleccionados. Presentamos el caso de un paciente pluripatológico con diagnóstico de empiema pulmonar izquierdo al que de manera accidental se le insertó un tubo torácico en el polo superior esplénico. La comorbilidad del paciente y la presencia de estabilidad hemodinámica abogaron por un tratamiento conservador mediante cateterización esplénica supraselectiva e introducción de cola quirúrgica en la retirada del tubo.

Palabras clave: Arteriografía. Traumatismo esplénico. Tubo de tórax.

\begin{abstract}
Inserting a chest tube is a therapeutic tool of great value not without complications. Our objective is to highlight a new treatment option using interventional radiological techniques that avoid the risks of surgery in selected patients. We present the case of a multi-pathological patient with a diagnosis of left pulmonary empyema who accidentally inserted a chest tube into the splenic superior pole. The comorbidities of the patient and the presence of hemodynamic stability advocated conservative treatment through supraselective splenic catheterization and the introduction of surgical glue in the withdrawal of the tube.
\end{abstract}

Key words: Arteriography. Splenic trauma. Chest tube.

\section{Introducción}

La colocación de un tubo torácico es una técnica habitual en la práctica clínica diaria, no exenta de complicaciones ${ }^{1}$, entre ellas las ocasionadas por la inserción del tubo dentro de la cavidad abdominal. Cada día más se tiende al tratamiento conservador si las características de la lesión y las condiciones del paciente lo permiten, evitando así el trauma de la cirugía.

\section{Caso clínico}

Presentamos el caso de un paciente varón de 84 años con antecedentes personales de hipertensión 
arterial, insuficiencia renal por nefroangioesclerosis y úlcera duodenal diagnosticado 3 meses antes de adenocarcinoma de colon sigmoides en estadio IV con masa metastásica pulmonar izquierda. Presentado el caso en comité multidisciplinario, se decide la colocación de una endoprótesis por tumoración estenosante e inicio de quimioterapia oral con capecitabina. El paciente acude a urgencias por fiebre de varios días de evolución y tras los estudios radiológicos se le diagnostica empiema pulmonar izquierdo. Se practica toracocentesis con salida de material purulento, por lo que se indica la colocación de un tubo de tórax. Ante la ausencia de débito por el tubo se realiza una tomografía computarizada toracoabdominal que muestra el mal posicionamiento del drenaje, con el tubo torácico atravesando el polo superior del bazo y llegando su extremo distal al hilio esplénico, sin signos de sangrado intraesplénico ni intraabdominal (Fig. 1).

Dado que el paciente se encuentra hemodinámicamente estable, sin dolor abdominal y sin descenso de la hemoglobina, se decide intentar un manejo conservador, para lo cual se realiza una arteriografía esplénica en la que se observa un pseudoaneurisma intraesplénico en el trayecto del tubo de tórax. A continuación se procede a cateterizar de manera supraselectiva la rama de la que depende y se emboliza con Onyx de forma segmentaria. Se deja colocado el catéter en esta rama y se cateteriza el tubo de tórax con un catéter $4 \mathrm{~F}$ que se lleva hasta la punta del mismo y se administra en su retirada Glubran ${ }^{\circledR}$ diluido 2/1 con lipiodol. Posteriormente se realiza un nuevo control angiográfico en el que ya no se observan pseudoaneurisma ni sangrado activo. Se colocan nuevamente dos tubos torácicos para drenaje del empiema. La evolución posterior es satisfactoria, manteniéndose asintomático desde el punto de vista abdominal, estable hemodinámicamente y sin anemización. Al quinto día se realiza una tomografía computarizada toracoabdominal de control (Fig. 2) en la que se objetivan cambios secundarios a embolización segmentaria de tercio medio del bazo y un pequeño infarto segmentario adyacente al área de embolización. Tras completar el tratamiento antibiótico de su proceso neumónico, el paciente es dado de alta para seguimiento ambulatorio.

\section{Discusión}

El riesgo de sepsis tras una esplenectomía y las potenciales complicaciones de una laparotomía en pacientes complejos hacen que cada vez se abogue más por un manejo conservador del trauma

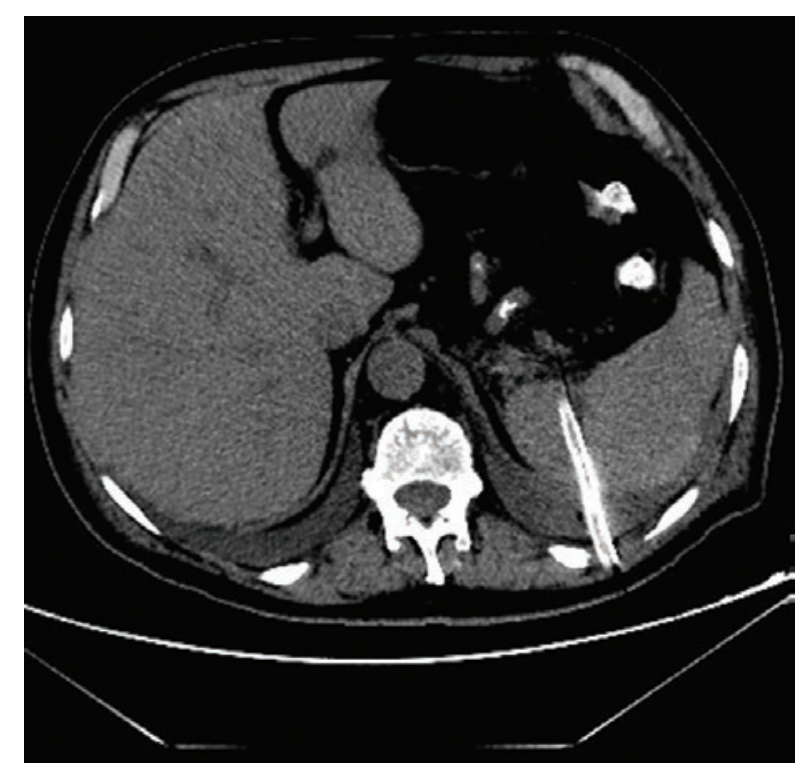

Figura 1. Tubo torácico insertado en bazo.

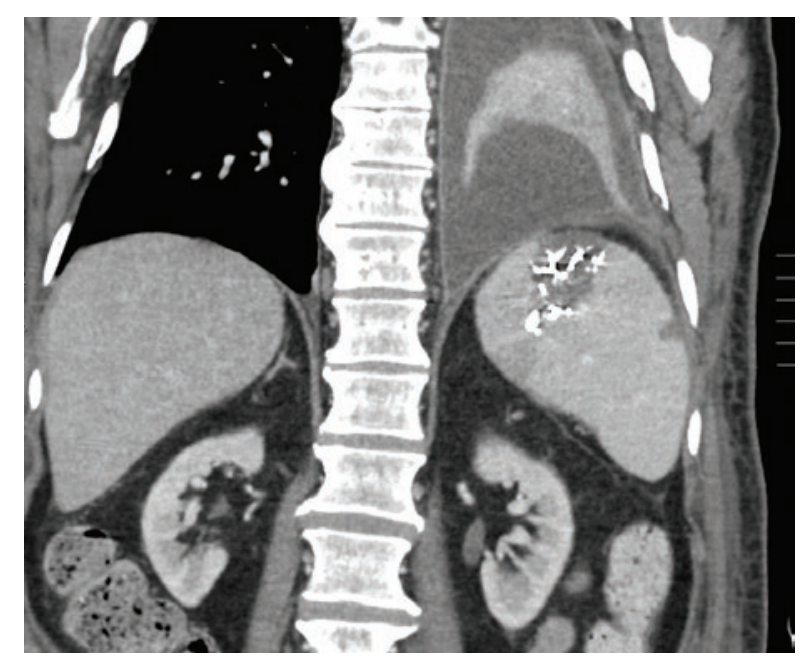

Figura 2. Control postembolización. Infarto esplénico segmentario.

esplénico. La tendencia actual va hacia un aumento de las intervenciones sobre la arteria esplénica para tratar diferentes condiciones clínicas ${ }^{2}$, siendo el traumatismo esplénico una de las más frecuentes. Las indicaciones para la embolización son fuga de contraste, pseudoaneurisma, lesiones de grado IV y V, y descenso continuo de la hemoglobina ${ }^{3,4}$; es condición imprescindible la estabilidad hemodinámica del paciente. Sin embargo, la embolización selectiva previa y la inyección de material hemostático en el trayecto durante la retirada del tubo de tórax es una técnica inusual, ya que en la revisión de la literatura solo hemos encontrado un caso que precisó angioembolización días después de la retirada del drenaje torácico, 
con desconocimiento de su mal posicionamiento esplénico previo $^{5}$, y otro caso de manejo conservador con mantenimiento del tubo torácico intraesplénico durante 2 semanas y su posterior retirada tras la formación de un trayecto fistuloso alrededor del tubo ${ }^{6}$.

Después de la embolización esplénica, sobre todo tras la embolización distal, pueden presentarse complicaciones ${ }^{7}$, tanto mayores (14\%; infarto esplénico, absceso esplénico, insuficiencia renal inducida por contraste, quiste esplénico sintomático) como menores $(34 \%$; derrame pleural izquierdo, mal posicionamiento o migración de coils, fiebre, pseudoaneurisma femoral). La mayoría de los pacientes pueden ser manejados de manera conservadora ${ }^{8}$. Por lo general, los infartos esplénicos habitualmente son asintomáticos y no precisan tratamiento, y los abscesos pueden requerir en ciertas ocasiones punción-drenaje radioguiado. La complicación más frecuente tras la embolización suele ser el resangrado, pero la mayoría de los pacientes son candidatos a una nueva angiografía y embolización con buenos resultados. La evidencia indica que la función inmunitaria está razonablemente bien conservada tras el tratamiento no quirúrgico con o sin angioembolización, por lo que no se recomienda la vacunación sistemática en estos pacientes.

\section{Conclusiones}

La proximidad del bazo al hemidiafragma izquierdo hace que pueda ser lesionado al colocar un tubo torácico, sobre todo si no se respeta el «triángulo de seguridad»": borde anterior del músculo dorsal ancho, borde lateral del músculo pectoral mayor y línea horizontal a nivel de la mamila; en ocasiones es necesario el uso de ecografía ${ }^{6}$ para la correcta inserción del drenaje torácico. Si a pesar de las medidas de prevención el bazo resulta lesionado, en caso de estabilidad hemodinámica puede plantearse, según la situación y las características del paciente, un manejo conservador que prevenga una futura sepsis posesplenectomía u otras posibles complicaciones de la cirugía exerética. La arteriografía con embolización supraselectiva y el relleno del trayecto con cola quirúrgica sintética en la retirada del tubo es una técnica factible que puede ser empleada a tal efecto.

\section{Agradecimientos}

Los autores agradecen su colaboración al personal facultativo y de enfermería de los servicios de cirugía del Hospital Universitario San Agustín y del Hospital
Universitario Central de Asturias, así como la ayuda recibida por el personal facultativo del servicio de radiodiagnóstico del Hospital Universitario Central de Asturias.

\section{Conflicto de intereses}

Los autores declaran que no existe conflicto de intereses.

\section{Financiamiento}

Los autores declaran que no se ha recibido ningún tipo de financiamiento para la realización de esta publicación.

\section{Responsabilidades éticas}

Protección de personas y animales. Los autores declaran que para esta investigación no se han realizado experimentos en seres humanos ni en animales.

Confidencialidad de los datos. Los autores declaran que han seguido los protocolos de su centro de trabajo sobre la publicación de datos de pacientes.

Derecho a la privacidad y consentimiento informado. Los autores han obtenido el consentimiento informado de los pacientes y/o sujetos referidos en el artículo. Este documento obra en poder del autor de correspondencia.

\section{Bibliografía}

1. Kesieme EB, Dongo A, Ezemba N, Irekpita E, Jebbin N, Kesieme C. Tube thoracostomy: complications and its management. Pulm Med. 2012;2012:256878.

2. Madoff DC, Denys A, Wallace MJ, Murthy R, Gupta S, Pillsbury EP, et al Splenic arterial interventions: anatomy, indications, technical considerations, and potential complications. Radiographics. 2005;25 (Suppl 1): S191-211.

3. Bhullar IS, Frykberg ER, Siragusa D, Chesire D, Paul J, Tepas JJ 3rd, et al. Selective angiographic embolization of blunt splenic traumatic injuries in adults decreases failure rate of nonoperative management. $J$ Trauma Acute Care Surg. 2012;72:1127-3.

4. Bhullar IS, Tepas JJ 3rd, Siragusa D, Loper T, Kerwin A, Frykberg ER. To nearly come full circle: nonoperative management of high-grade IV-V blunt splenic trauma is safe using a protocol with routine angioembolization. J Trauma Acute Care Surg. 2017;82:657-64.

5. Kelly $M D$, Jones $L$. latrogenic penetrating splenic injury treated by embolization. ANZ J Surg. 2009;79:393-4.

6. Ohtaka K, Hase R, Chiba R, Miyasaka M, Sato S, Shoji Y, et al. Noninvasive management for iatrogenic splenic injury caused by chest tube insertion: a case report. Clin Case Rep. 2016;4:1157-60.

7. Ekeh AP, Khalaf S, llyas S, Kauffman S, Walusimbi M, McCarthy MC. Complications arising from splenic artery embolization: a review of an 11-year experience. Am J Surg. 2013;205:250-4.

8. Haan JM, Biffl W, Knudson MM, Davis KA, Oka T, Majercik S, et al.; Western Trauma Association Multi-Institutional Trials Committee. Splenic embolization revisited: a multicenter review. J Trauma. 2004;56:542-7.

9. Laws D, Neville E, Duffy J; Pleural Diseases Group, Standards of Care Committee, British Thoracic Society. BTS guidelines for the insertion of a chest drain. Thorax. 2003;58 (Suppl 2):ii53-9. 